Original article

\title{
Degeneration of spinal ganglion and segmental apparatus of the spinal neurons in sciatic nerve injury: an experimental study
}

\author{
Vladimir S. Tolkachev, Sergey P. Bazhanov, Olga V. Matveeva, Galina A. Korshunova, Stanislav D. Shuvalov, \\ Valdimir Yu. Ulyanov, Vladimir V. Ostrovskij
}

Saratov State Medical University, Saratov, Russia

Received 25 January 2021, Revised 20 November 2021, Accepted 30 November 2021

(c) 2021, Russian Open Medical Journal

Abstract: Objective - To investigate the extent of degenerative changes in neurons of spinal ganglion and segmental apparatus in various injuries to sciatic nerve in the experiment on white rats.

Material and Methods - The research involved 40 white non-pedigree male rats distributed among four groups. The animals of Group 1 $(n=10)$ underwent the compression of nerve trunks with Mosquito clamp forceps for 15 minutes. In Group 2 ( $n=10$ ), the animals had their nerve trunks ligated; and in Group 3, they had their nerves completely transected in their middle thirds. The separate group of control animals $(n=10)$ suffered no damage to their sciatic nerves. Spinal cords and spinal ganglia at L4-L6 level were the material for histopathological examination. We calculated the number (percent) of degenerated neurons in spinal cords and spinal ganglia at the affected sides on Day 30, and compared them to those at the intact sides.

Results - The number (percent) of degenerated neurons in spinal cord and spinal ganglion, expressed as Me (Q1; Q2), constituted 2.52\% $(1.92 ; 2.74)$ and $3.75 \%(2.37 ; 4.74)$ in Group $1,9.27 \%(9.03 ; 9.94)$ and $16.74 \%(16.01 ; 18.22)$ in Group $2,25.59 \%(24.36 ; 26.29)$ and $31.94 \%$ $(31.44 ; 33.03)$ in Group 3, respectively. Depending on the number (percent) of degenerated neurons, we classified three grades of change manifestation: mild (Group 1), medium (Group 2), and severe (Group 3). No degenerated neurons were found in the control animals.

Conclusion - The compression, ischemic exposure on the sciatic nerve, and complete anatomical transection of its trunk resulted in Wallerian degeneration, as well as degeneration of segmental apparatus in spinal cord neurons.

Keywords: trauma, nerve, spinal cord, degeneration.

Cite as Tolkachev VS, Bazhanov SP, Matveeva OV, Korshunova GA, Shuvalov SD, Ulyanov VYu, Ostrovskij VV. Degeneration of spinal ganglion and segmental apparatus of the spinal neurons in sciatic nerve injury: an experimental study. Russian Open Medical Journal 2021; 10: e0424.

Correspondence to Vladimir S. Tolkachev. Address: N.G. Chernyshevskogo str., 148, Saratov, 410002, Russia. Phone: +79873284054. Email: vladimir.tolkache@yandex.ru.

\section{Introduction}

The so-called Wallerian degeneration is a well-studied phenomenon described in detail in both domestic and foreign published sources. It features changes in the distal segments of the damaged peripheral nerves and denervated muscles a few hours after trauma [1]. In recent years, medical examiners and pathophysiologists suggested that these pathological changes may proceed the other way around and involve the proximal segment of the nerve, as well as spinal ganglion and neurons in the segmental apparatus of the spinal cord $[2,3]$.

While studying the damage to peripheral nerves, many researchers considered the functional recovery of the limbs insufficient even after various combination therapy methods have been employed. This may be caused by neuronal degeneration due to disruption of both structural and functional continuities of the neural circuit [1, 3-5].

It should be noted that the mechanisms of degeneration process in spinal cord neurons in conditions of damaged peripheral nerve trunks is insufficiently studied. This is due to the fact that the integrity of all segments in the neural circuit is difficult to trace, and finding the changes in the segmental apparatus of human spinal cord is problematic [4, 5].

Such phenomena are studied on experimental animals. The most commonly used models for peripheral nervous system damage are transection, compression, and ligation of the sciatic nerve trunks in rats. The changes in spinal cord neurons under the damage to sciatic nerves in white rats are evaluated via the examination at the L4-L6 level $[6,7]$.

It is worth noting that the degeneration of spinal cord and spinal ganglion neurons is not studied well enough, since there is no reliable data on the minimal possible damage to peripheral nerve leading to the development of such changes.

This research was aimed at detecting the extent of neurodegenerative changes at epicone and cone (L4-L6 segments) levels in various injuries of sciatic nerves in the experiment on white rats. 


\section{Material and Methods}

\section{Laboratory animals}

The research involved 40 white non-pedigree male rats with a body weight of 180-240 g, kept in the vivarium and provided with free access to food and water. The experiments were conducted at the Federal State Budgetary Institution of Higher Education, Vavilov State Agricultural University of Saratov, the Russian Federation Ministry of Agriculture. The research was approved by the Ethics Committee and complied with relevant regulations (Directive No. 755 of the Russian Federation Ministry of Healthcare of August 12, 1977, On Measures for Further Improvement of the Organizational Forms of Working with Experimental Animals; Directive No. 266 of the Russian Federation Ministry of Healthcare of June 19, 2003, On Adoption of the Regulations for Laboratory Practices) and the 1983 revision of the Declaration of Helsinki.

\section{Surgical procedures}

Surgical procedures and other invasive interventions were performed under general anesthesia with $0.1 \mathrm{~mL} / \mathrm{kg}$ of zoletil and $0.1 \mathrm{~mL} / \mathrm{kg}$ of xylazine at a dose of $1 \mathrm{mg} / \mathrm{kg}$ of the rat weight, administered via intramuscular injections. Sciatic nerves were isolated from the middle thirds of the femurs, using microsurgical instruments, and then the animals were distributed among the groups. In animals of Group $1(n=10)$, the nerves were compressed for 15 minutes with $12.5 \mathrm{~cm}$ long, $0.02 \mathrm{~kg}$ Halsted-Mosquito clamp forceps, with their closed ramus $4 \mathrm{~mm}$ wide and a retention pattern formed with grooves perpendicular to the instrument axis (simulation of compression and ischemic injury). In animals of Group $2(n=10)$, the nerves were ligated on the same level with nonabsorbable filament Premilen UPS $6 / 0$. The applied compression ratio caused no deformity to the visible contours of the nerve trunks, and it was left for 30 days. In animals of Group 3 $(n=10)$, sciatic nerves were isolated and completely transected with a $140 \mathrm{~mm}$ long ophthalmic general operating knife at the level of the middle third of the femur without their further neurorrhaphy. We performed no surgical interventions on sciatic nerves of the control animals $(n=10)$ (with no surgical wounds in the projection of the nerve trunks). After surgical procedures, the new soft tissue defects were sutured with an atraumatic UPS 5/0 suture. The animals (Groups 1-3) were kept in individual containers and provided with a proper care as well as with access to sufficient amounts of food and water. We monitored the changes in the condition of operated animals throughout the experiment.

\section{Pathomorphological examination}

The rats were sacrificed by intramuscular injections of Lysthenon on Day 30. At that point, we performed laminectomies, using microsurgical instruments, and removed the distal fragments of spinal cords with spinal ganglia. The biomaterial was preserved with a $10 \%$ solution of neutral formalin and hydrated through an increasing series of alcohol (70\% alcohol I - $2 \mathrm{~h}, 70 \%$ alcohol II -3 h, $80 \%$ alcohol I -3 h, $80 \%$ alcohol II -4 h, $96 \%$ alcohol I -3 h, $96 \%$ alcohol II -4 h, $96 \%$ alcohol III -2 h). Then, the material was dehydrated (chloroform I - $30 \mathrm{~min}$, chloroform II - $30 \mathrm{~min}$, chloroform III - $30 \mathrm{~min}$, chloroform IV - $30 \mathrm{~min}, 1: 1$ chloroform and paraffin mixture $-2 \mathrm{~h}$ ). The biomaterial was then paraffinembedded (paraffin I - $1.5 \mathrm{~h}$, paraffin II $-19 \mathrm{~h}$ ), and 5-7 $\mu \mathrm{m}$ thick sections were cut by the RMD 3000 semi-automatic rotary microtome and stained with hematoxylin and eosin. The samples were studied using the Olympus CX31 microscope with a VIDI-CAM module. We calculated the number of degenerated neurons, using the calibrated ocular grid, considering the number of spinal cord neurons on the intact side as 100 percent.

When studying histologic sections, we paid special attention to the number of vessels full of blood, the number and condition of ganglion cells (size and shape of the cells, presence of nucleoli), and stromal edema. The arrangement of pseudounipolar neurons, their shape, and size, as well as the presence of nucleoli, were taken into account when studying histological specimens. All these features are indicative of degenerative changes in ganglion cells.

\section{Statistical analyses}

We used Statistica 10.0 for Windows (SPSS version 23) software package for statistical processing of our findings. Since the initial check with Shapiro-Wilk and Kolmogorov-Smirnov tests demonstrated non-normal distributions, we employed the distribution-free methods to compute the median and interquartile ranges: Me (Q1; Q3). The differences between the analyzed groups were assessed with Mann-Whitney $U$ test, Kruskal-Wallis test, and Wilcoxon rank-sum test. They were considered statistically significant at $p \leq 0.05$.

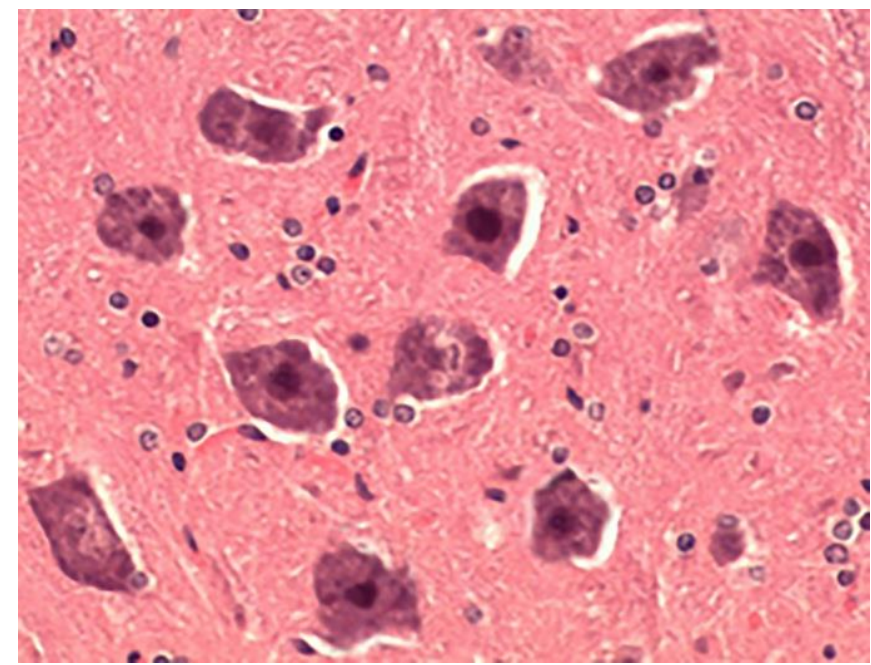

Figure 1. Intact neurons (control), hematoxylin-eosin stained, 400x.

Table 1. Statistical characteristics of degenerated neurons in spinal ganglion and segmental apparatus of the spinal cord

\begin{tabular}{|c|c|c|c|c|}
\hline Levels & Control & Group 1 & Group 2 & Group 3 \\
\hline $\begin{array}{l}\text { Degenerated spinal cord neurons at } \\
\text { the epicone and cone levels }\end{array}$ & $152(143.75 ; 157.50)$ & $\begin{array}{c}152(146.75 ; 155.5) \\
\mathrm{P}^{\Delta}=0.0051 \mathrm{P}_{1-0}^{\circ}=0.969\end{array}$ & $\begin{array}{c}137(134.0 ; 141.75) \\
\mathrm{P}^{\Delta}=0.0051 \mathrm{P}_{1-2}^{\circ}=0.011\end{array}$ & $\begin{array}{c}113.50(110.0 ; 116.0) \\
\mathrm{P}^{\Delta}=0.0051 \mathrm{P}_{2-3}^{\circ}=0.00001 \mathrm{P}_{1-3}^{\circ}=0.00001\end{array}$ \\
\hline $\begin{array}{l}\text { Degenerated spinal ganglion } \\
\text { neurons }\end{array}$ & $109.5(106.75 ; 122.25)$ & $\begin{aligned} 104.50(101.75 ; 116.25) \\
\mathrm{P}^{\Delta}=0.0744 \mathrm{P}_{1-0}^{\circ}=0.163\end{aligned}$ & $\begin{array}{c}91(89 ; 103.25) \\
\mathrm{P}^{\Delta}=0.0051 \mathrm{P}_{1-2}^{\circ}=0.0089\end{array}$ & $\begin{array}{c}75.5(73 ; 85.25) \\
\mathrm{P}^{\Delta}=0.0051 \mathrm{P}_{2-3}^{\circ}=0.0004342 \mathrm{P}_{1-3}^{\circ}=0.00018\end{array}$ \\
\hline
\end{tabular}

$\mathrm{P}^{\Delta}-p$-value to compare healthy and affected sides via the Wilcoxon rank-sum test; $\mathrm{P}_{j-i}^{\circ}-p$-value to compare differences in pathologies among $j$-th and $i$-th groups via the Mann-Whitney U test. 


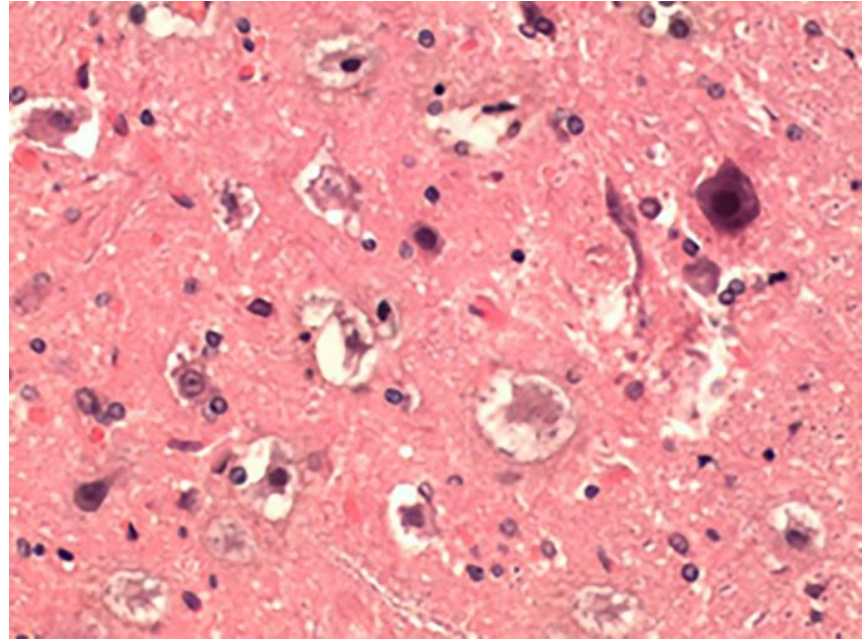

Figure 2. Degenerated neurons (Group 2), hematoxylin-eosin stained, $400 x$.

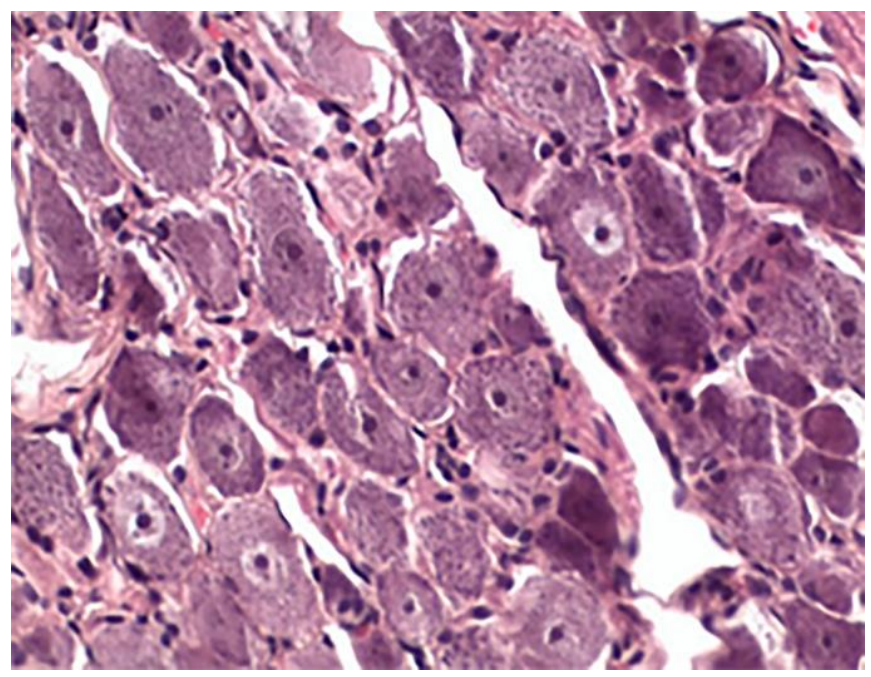

Figure 3. Intact ganglion (control), hematoxylin-eosin stained, 400x.

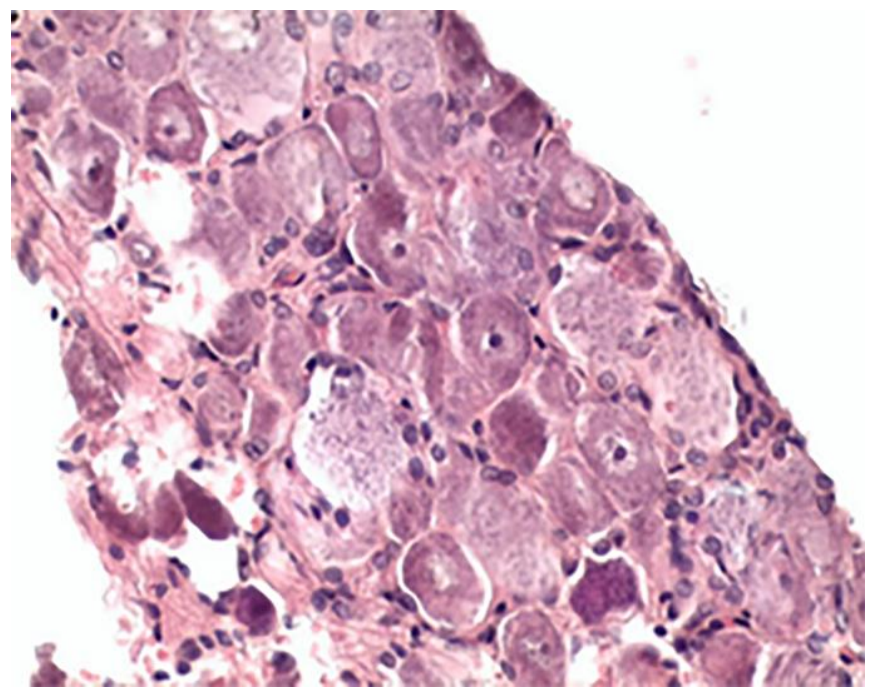

Figure 4. Degenerated spinal ganglion (Group 3), hematoxylin-eosin stained, $400 \times$.

\section{Results}

The investigation of histological specimens revealed degenerative changes of neurons in all experimental groups (1-3).

The homogeneity in all analyzed groups ( $p=0.504$ for spinal ganglions, $p=0.618$ for spinal cords) at the level of the intact limb was established with the Kruskal-Wallis test.

The investigation of the degenerative changes in spinal ganglions involved the Kruskal-Wallis testing of homogeneity hypothesis in the groups of animals with affected limb, and revealed the differences between the groups $(p<0.00001)$. The pairwise comparisons resulted in statistically significant differences between the controls vs. Group 2, control group vs. Group 3, Group 1 vs. Group $3(p<0.00001)$. The comparison of spinal ganglions in healthy and affected sides in Group 1 revealed no differences at $p=0.074$ (the Wilcoxon rank-sum test), but since the sample number was small $(n=10)$ and confidence level was close to significant we propose that these differences actually exist. The differences were also determined for Group 2 $(p=0.0051)$ and Group $3(p=0.0051)$.

The z-score for multiple comparisons revealed the statistically significant differences only in Group $3(p<0.00001)$, as compared with other groups. The differences between other groups were statistically insignificant, although within the groups, the differences between affected and healthy limbs were obvious.

Therefore, Group 1 presented the small number (percent) of degenerated nerve cells that we regarded as mild damage to the spinal cord and ganglion neurons. The changes in spinal cords in Group 2 were regarded as medium damage, and Group 3 represented the severe grade of neuron degeneration. No degenerative changes were observed in the control rats.

It is worth noting that there were more degenerated neurons in spinal ganglions, as compared with those in spinal cords: such difference was found in Groups 2 and 3 with intragroup comparisons of the proportionate factor (percent) for degenerated neurons (the Wilcoxon rank-sum test $\mathrm{p}=0.0051$ ).

The true Wilcoxon rank-sum and Mann-Whitney $U$ test $p$ values for each analyzed group are presented in the Table 1.

As the study of histological specimens in the intact animals demonstrated, the multipolar neurons kept their former shape and size with the central position of nucleoli. The neurocytes were mainly localized as aggregates or stretched columns with individual small full-blooded vessels (Figure 1), which corresponded to the norm.

Even though some neurons in the experimental groups (1-3) remained unchanged, they suffered moderate pericellular edema and pyknosis, formed microcysts, featured multiple full-blooded vessels; and their nerve cell bodies shrank, changed, and lost nuclei (Figure 2).

Pseudounipolar neurons in the spinal ganglia of the control animals were sitting tight, their form and size identical (Figure 3), whereas in the spinal ganglia of the experimental animals (Group 1-3), the cell bodies changed their shape and size and lacked nuclei (Figure 4).

\section{Discussion}

There were studies confirming the retrograde degeneration in the spinal cord and spinal ganglion neurons with peripheral nerve injury. In these publications [2-3], the authors presented the 
evaluation results concerning the changes in the lesions and restoration of the spinal cord and spinal ganglion neurons at various time points, based on the morphological examination of motoneuron populations, forming sciatic nerves at both sides of the rats. It was established that after axotomy at various time points of post-traumatic period the ratio of different motoneurons is variable, but it returns to the initial values by Day 300 , even though the number of neurons within the population decreases. However, no data on the intensity of degenerative changes in nervous structures, depending on the severity of the sciatic nerve damage, was previously presented.

The results of other studies $[8,9]$ exhibited morphofunctional cell changes, including those of synaptic nature. They took place in motoneurons of rat spinal cords after sciatic nerve injury, and featured the reduction in signals for synaptic adhesion molecules. The authors also established that peripheral axotomy triggered retrograde degeneration, distal in relation to the lesion, and was accompanied by the loss of synapses (synaptic removal) from the surface of damaged spinal cord motoneurons, affected by reactive astrocytes and microglia (total removal of axon collaterals) in neuroinflammatory response. The authors also presented the data on the alteration of spatial arrangement of neurons and glia cells in anterior cornu. However, no data on the development of retrograde degeneration with respect to the severity of alterations in spinal cord nervous structures was presented.

The findings of another study [10] were related to the investigation of synaptic plasticity of motoneurons after axotomy, depending of general mechanisms in charge of trauma severity, because synaptic plasticity defined the potential errors of targeting the peripheral sensory and motor axons. The axons turned the afferent cohesion in spinal cord's maladaptive and reduced synaptic activity. However, the author provided no data on the severity of damage to spinal ganglion and spinal cord neurons, depending on the severity of damage to sciatic nerve.

In [11], the retrograde degeneration was confirmed with changes in muscular activity in rats (based on electroneuromyography data) after sciatic nerve transections. It featured disappearance of soleus and gastrocnemius H-reflexes, and repression of direct $M$-responses to posterior tibial nerve stimulation. This study has also drawn no conclusion about the severity of changes in morphology of spinal cord neurons, depending on the severity of sciatic nerve injury.

It was the lack of reliable conclusions in above-listed papers on the severity of retrograde degeneration in the spinal cord and ganglion that makes our research relevant in view of the necessity to evaluate this pathological process, depending on the severity of mechanical damage to the sciatic nerve. We introduced the concept of proportional factor of the injury to both spinal cord and ganglion that enabled reliable determination of the damage severity to peripheral nerves, and quantitative evaluation of the efficacy of neuroregeneration and neuroplasticity as components of neuronal remodeling in the course of neuroprotective therapy.

\section{Conclusion}

Histomorphological analysis of various lesions imposed upon experimental animals revealed that the damage to the peripheral nerve has caused not just the orthograde degeneration (sensu Waller) but the retrograde degeneration as well, featured by the degeneration of neurons in the spinal ganglia and involved segments of the spinal cord. While short-term compression of the nerve caused mild damage to the spinal ganglion and spinal cord neurons, long-term compression resulted in the medium changes, and nerve transection has led to severe damage.

\section{Conflict of interest}

This research was a part of the Public Procurement No. 121032300173-9, Designing Medical Decision-Making Support System in Combined Treatment of Peripheral Nervous System Injuries with Electrical Neuromodulation Methods, performed at the Scientific Research Institute of Traumatology, Orthopedics and Neurosurgery, Federal State Budgetary Institution of Higher Education V.I. Razumovsky State Medical University of Saratov, the Russian Federation Ministry of Healthcare.

\section{References}

1. Bersnev VP, Kockin GS, Izveckova TO. Practical Guide to Neurosurgery: in 2 volumes. Vol. 1. Saint Petersburg: RNHI n.a. AL Polenov. 2009; 291 p. https://search.rsl.ru/ru/record/01004639672.

2. Blagova NV. Normal Morphology of Spinal Cord Motor Neurons and Transection of the Sciatic Nerve in Adult Rats. PhD thesis abstract. Saransk, 2011; 23 p. https://www.elibrary.ru/item.asp?id=30359943.

3. Timofeeva LB. Normal Morphology of Spinal Ganglion and Transection of the Sciatic Nerve in Adult Rats. PhD thesis abstract. Saransk, 2011; 17 p. v https://www.elibrary.ru/item.asp?id=19360062.

4. Puanhvuan D, Chumnanvej S, Wongsawat Y. Linear model of peripheral nerve after surgical manipulation: Preliminary report in animal study and model shift. Annu Int Conf IEEE Eng Med Biol Soc 2013; 2013: 4973-4976. https://doi.org/10.1109/embc.2013.6610664.

5. Berg A, Zelano J, Cullheim S. Netrin G-2 ligand mRNA is downregulated in spinal motoneurons after sciatic nerve lesion. Neuroreport 2010; 21(11): 782-785. https://doi.org/10.1097/wnr.0b013e32833cadd8.

6. Savastano LE, Laurito SR, Fitt MR, Rasmussen JA, Gonzalez Polo V, Patterson SI. Sciatic nerve injury: a simple and subtle model for investigating many aspects of nervous system damage and recovery. $J$ Neurosci Methods 2014; 227: 166-180. https://doi.org/10.1016/j.jneumeth.2014.01.020.

7. Gordon T, Borschel GH. The use of the rat as a model for studying peripheral nerve regeneration and sprouting after complete and partial nerve injuries. Exp Neurol 2017; 287(Pt 3): 331-347. https://doi.org/10.1016/j.expneurol.2016.01.014.

8. Berg A, Zelano J, Pekna M, Wilhelmsson U, Pekny M, Cullheim S. Axonal regeneration after sciatic nerve lesion is delayed but complete in GFAP- and vimentin-deficient mice. PLoS One 2013; 8(11): e79395. https://doi.org/10.1371/journal.pone.0079395.

9. Rashidiani-Rashidabadi A, Heidari MH, Sajadi E, Hejazi F, Fathabady FF, Sadeghi $Y$, et al. Sciatic nerve injury alters the spatial arrangement of neurons and glial cells in the anterior horn of the spinal cord. Neural Regen Res 2019; 14(10): 1833-1840. https://doi.org/10.4103/16735374.257539.

10. Alvarez FJ, Rotterman TM, Akhter ET, Lane AR, English AW, Cope TC. Synaptic plasticity on motoneurons after axotomy: A necessary change in paradigm. Front Mol Neurosci 2020; 13: 68. https://doi.org/10.3389/fnmol.2020.00068.

11. English AW, Chen Y, Carp JS, Wolpaw JR, Chen XY. Recovery of electromyographic activity after transection and surgical repair of the rat sciatic nerve. J Neurophysiol 2007; 97(2): 1127-1134 https://doi.org/10.1152/jn.01035.2006.

\section{Authors:}

Vladimir S. Tolkachev - MD, Junior Research Assistant, Department of Innovative Projects for Neurosurgery and Vertebrology, Scientific Research Institute of Traumatology, Orthopedics and Neurosurgery, Saratov State Medical University n.a. V.I. Razumovsky, Saratov, Russia. https://orcid.org/0000-0001-6580-4403. 
Sergey P. Bazhanov - MD, DSc, Head of the Department of Innovative Projects for Neurosurgery and Vertebrology, Scientific Research Institute of Traumatology, Orthopedics and Neurosurgery, Saratov State Medical University n.a. V.I. Razumovsky, Saratov, Russia. https://orcid.org/00000001-9474-9095.

Olga V. Matveeva - MD, PhD, Pathologist, Department of Laboratory Diagnostics, Scientific Research Institute of Traumatology, Orthopedics and Neurosurgery, Saratov State Medical University n.a. V.I. Razumovsky, Saratov, Russia. http://orcid.org/0000-0002-3536-2744.

Galina A. Korshunova - MD, PhD, Senior Research Assistant, Department of Innovative Projects for Neurosurgery and Vertebrology, Functional Diagnostics Doctor in the Functional Diagnostics Department, Scientific Research Institute of Traumatology, Orthopedics and Neurosurgery, Saratov State Medical University n.a. V.I. Razumovsky, Saratov, Russia. https://orcid.org/0000-0003-3648-0141.

Stanislav D. Shuvalov - Resident Physician, Department of Neurosurgery, Saratov State Medical University n.a. V.I. Razumovsky, Saratov, Russia. https://orcid.org/0000-0002-8095-9398.

Valdimir Yu. Ulyanov - MD, DSc, Associate Professor, Deputy Director on Science and Innovations, Scientific Research Institute of Traumatology, Orthopedics and Neurosurgery, Saratov State Medical University n.a. V.I. Razumovsky, Saratov, Russia. https://orcid.org/0000-0002-9466-8348.

Vladimir V. Ostrovskij- MD, DSc, Director, Scientific Research Institute of Traumatology, Orthopedics and Neurosurgery, Saratov State Medical University n.a. V.I. Razumovsky, Saratov, Russia. https://orcid.org/00000002-8602-2715. 